\title{
Influence of viscosity, surface tension, and inclination angle on motion of long bubbles in closed tubes
}

\author{
By E. E. ZUKOSKI \\ Guggenheim Jet Propulsion Center, California Institute of Technology, \\ Pasadena, California
}

(Received 28 September 1965)

An experimental study has been made of the motion of long bubbles in closed tubes. The influence of viscosity and surface tension on the bubble velocity is clarified. A correlation of bubble velocities in vertical tubes is suggested and is shown to be useful for the whole range of parameters investigated. In addition, the effect of tube inclination angle on bubble velocity is presented, and certain features of the flow are described qualitatively.

\section{Introduction}

The propagation rate of a long bubble through a vertical tube closed at the upper end has been studied by a number of investigators (Barr 1926; Davies \& Taylor 1950; Dumitrescu 1943; Goldsmith \& Mason 1962; Harmathy 1960; Hattori 1935), and the general features of the flow are known. The purpose of the present study is to determine, in a more precise manner, the influence on bubble propagation-rate of surface tension, viscosity, and tube inclination for the flow régime in which surface-tension effects are important.

Previous studies have been restricted to the investigation of propagation rates of bubbles in vertical tubes. For this geometry, and for fluids which wet the walls, the bubble is axisymmetric and the light material moves up the centre of the tube in a long, ogival-shaped finger. The heavier material moves around the nose of the bubble and falls in a thin sheet attached to the tube wall. The velocity and bubble nose geometry have been found to be independent of the length of the bubble for lengths greater than a few tube radii (Barr 1926; Hattori 1935).

The velocity of the bubble in very large diameter, vertical tubes has been shown to be proportional to $(g a)^{\frac{1}{2}}$, where $g$ is the local gravitational acceleration and $a$ is the radius. However, when the tube size is reduced sufficiently, the velocity decreases faster than $(a)^{\frac{1}{2}}$, and for tubes of small enough diameter, bubble motion ceases completely. In this range of tube diameters, surface tension is important, and the critical size for no motion is fixed by capillary effects. As the tube size and velocity decrease, the velocity also becomes a function of the viscosity of the fluids involved.

Although this discussion has been restricted to bubbles rising through a more dense medium, similar statements hold concerning the motion of drops of a fluid falling through a lighter medium. 
Theoretical treatments of the problem for predicting the motion of the bubble have been carried out for the special case of a vertical tube with the additional restrictions that either both viscous and surface-tension effects are negligible (Davies \& Taylor 1950; Dumitrescu 1943), or that viscous effects dominate (Goldsmith \& Mason 1962). The approximate results of Dumitrescu lead to satisfactory prediction of bubble velocity and geometry for the first set of assumptions. The agreement of the more approximate analysis of Davies \& Taylor with experimental results depends on the selection of an arbitrary radius at which certain boundary conditions are satisfied. Unfortunately, the predicted velocity depends strongly upon which radius is selected.

The analysis of Goldsmith \& Mason gives a framework for analysis of details of the fluid motion for the viscous case, but does not lead to a prediction of parameters such as bubble velocity or film thickness. None of these theoretical analyses form a basis for extension of the problem to include surface-tension effects or the effect of tube inclination.

In addition to these theoretical analyses, a great deal of experimental work has been done on bubble motion in vertical tubes, and a portion of this work has been recently reviewed in a paper by Harmathy. The early interest in bubble motion in vertical tubes resulted from the use of such devices for the measurement of the viscosity and surface tension of fluids. The measurement of viscosity depends on the fact that under certain conditions the velocity of a gas bubble in a vertical tube is inversely proportional to the viscosity of the fluid in the tube; the work of Barr was directed toward a better understanding of this device. The surface-tension measurement was made by determining the tube diameter at which no bubble velocity could be discerned. A theoretical treatment was then used to relate the surface tension to the square of the tube diameter at which the bubble propagation ceased. This device was proposed and investigated by Hattori.

The experimental work of these investigators, and most of the subsequent work, has been directed either toward the régime in which bubble velocity is small and hence viscous effects are important, or toward the régime in which neither viscous nor surface-tension effects are important. The transition between these régimes and the effects of surface tension in general have not been completely clear.

The material presented in this paper describes an experimental investigation of the effects of viscosity, surface tension, and tube inclination on the velocity of long bubbles. The range of parameters used in this investigation made it possible to cover flow régimes enabling the full range of influence of viscosity and surface tension to be investigated.

\section{Experimental technique}

Tests were carried out with a number of fluid combinations and with a number of tube sizes. The fluids used included water, carbon tetrachloride, mercury, glycerine, ethyl alcohol, and mixtures of the latter two with water. In most cases, the fluid properties were taken from tabulated values and were not measured by the author. However, the viscosity of the glycerine and glycerine-water mixtures was measured. 
A number of tubes were used with diameters lying in the range between 0.5 and $17 \cdot 8 \mathrm{~cm}$, and most of the tubes were standard soft-glass drawn tubing about $1 \mathrm{~m}$ long. Lucite tubing was also used in a few cases to check the influence of contact angle on the propagation of the bubble. It was found that tube material had no influence on the flow when the diameter was greater than $2 \mathrm{~cm}$, since for these diameters the tube wall was always wetted a distance of several tube diameters downstream of the nose of the bubble. Thus, for these large-diameter tubes, surface properties of the wall played no part in the problem. Tube diameters were obtained from measurements of the tube volume for a measured length. The glass tubes were prepared for use by thoroughly washing with soap and water followed by rinses of distilled water and $100 \%$ ethyl alcohol. After drying in air, the tubes were ready for use.

Three techniques were used for velocity measurements. The first technique was used primarily for air-bubble measurements. A tube, closed at one end, was filled with the desired fluid, and the open end was then placed under the surface of the same fluid in a beaker. The inclination of the tube was fixed and the bubble was formed by moving the beaker to expose the open end of the tube to the air. The time required for the bubble to move a measured distance along the tube was then determined and was used to calculate the velocity.

The second technique was used for the investigation of bubbles or drops of liquids moving through other liquids. A vertical tube closed at the lower end was completely filled with the two fluids in the equilibrium condition, i.e. the lighter on top, and with the bubble or droplet column considerably shorter than the primary column. The tube was then closed and suddenly placed at the desired inclination with the heavy fluid at the higher end, and the subsequent motion of the heavy droplet was observed.

Finally, when measurements were made in the $17 \cdot 8 \mathrm{~cm}$ diameter plastic tube, rubber balloons were placed at the lower end of the tube which had previously been filled with water. They were inflated to the desired volume of air and then ruptured to form the air bubble.

Stop watches were used to determine the time required for the bubbles to move a fixed distance. Some time measurements were also made with photocells used to trigger an electronic counter. The two methods agreed within a few per cent. In a number of cases, the tubes were long enough to permit time-of-transit measurements at several distances from the outlet end of the tube. These measurements indicated that the bubble velocity reaches a steady value within 5 or 10 radii of the tube exit and remains constant thereafter. The only exceptions to this result occurred when the tubes were horizontal or almost horizontal. These exceptions are described later in the paper.

Velocities were calculated from the measured values of transit times and distances. In all cases, the reported speeds represent averages of at least five tests which had a variation of less than $\pm 5 \%$ from the average value. The overall uncertainty of velocity determination was judged to be less than $\pm 3 \%$.

In some of the work, it was convenient to use bubbles of finite length rather than to let the tube drain completely. Hence, a careful check of the effect of bubble length on bubble velocity was carried out. The results showed that the 
propagation velocity is independent of length as long as the volume of the bubble corresponds to a cylinder with the tube radius and a length of 3 tube radii. As the effective length is reduced from three radii to one, the bubble velocity goes through a weak minimum and then starts to increase above the value obtained for long bubbles. In all cases reported here, the effective bubble length was kept greater than four tube radii.

When photographs were taken of the bubbles, the tubes were placed in a container filled with the test fluid and the bubbles were viewed through a plane window in the container. Measurements made on the photographs showed that distortion due to different indices of refraction of the tube wall and the various fluids was negligible.

\section{Analytical considerations}

Before presenting the experimental results it is of interest to discuss briefly the dimensionless parameters used in their presentation. The parameters used here were selected after examining the differential equations and boundary conditions for the motion of a bubble of inviscid material rising through a viscous primary fluid in a vertical tube. The factors used to make the variables non-dimensional were selected to give the minimum number of parameters in the dimensionless statement of the problem. The tube radius $\alpha$ and a velocity given by

$$
U_{0} \equiv[g a(\Delta \rho / \rho)]^{\frac{1}{2}}
$$

were selected to normalize the co-ordinates and velocities, and the normalized pressure was taken to be $\left[\left(P-\rho_{b} g Z\right) / \Delta \rho g a\right]$. In this paper, $g$ is the gravitational acceleration, and $\mu$ and $\rho$ are the viscosity and density of the primary fluid into which the bubble is propagating; $\Delta \rho$ is the absolute value of the density difference between the primary and bubble fluid, $\sigma$ is the interfacial tension, and $\rho_{b}$ is the density of the bubble fluid. The $Z$ axis is parallel to the tube axis and has its origin at the nose of the bubble.

When the groups given above are used to normalize the variables in the differential equations and boundary conditions, the statement of the mathematical problem only depends on two parameters. These are a Reynolds number of the form $\left(\rho U_{0} a / \mu\right) \equiv R e_{0}$ and a surface-tension parameter, $\left[\sigma / \Delta \rho g a^{2}\right] \equiv \Sigma$. Thus, this analysis indicates that when $R e_{0}$ and $\Sigma$ have equal values for different systems, the normalized velocity field and bubble geometry for these systems will be similar.

In reporting the experimental results, the normalized velocity is chosen as $w_{b}=\left(W_{b} / U_{0}\right)$. The Reynolds number of the bubble based on the bubble velocity, $R e \equiv\left(\rho W_{b} a / \mu\right)=\left(R e_{0}\right)\left(W_{b} / U_{0}\right)$, is used to characterize the viscous effects, and the surface-tension parameter $\Sigma$ is used as a measure of the surface-tension influence. $\dagger$

The Reynolds number used here is the conventional one, and the surfacetension parameter is the ratio of the surface-tension forces per unit area, $\sigma / a$, to the dynamic pressure of the flow, $\rho U_{0}^{2}$. Note that the radius of curvature used in

$\dagger$ Note that $\Sigma$ is the reciprocal of the more commonly used Bond number. 
evaluating the surface-tension forces is the tube radius $a$. In some cases, the important radius of curvature is much smaller than $a$, and in these cases $\Sigma$ is a poor measure of the relative magnitude of the surface-tension influence.

\section{Experimental results}

Typical bubble shapes for a tube with $a=0.44 \mathrm{~cm}$ are shown in figure 1 (plate 1). The bubbles are viewed along a normal to the plane of inclination of the tube and the inner tube walls are indicated by the arrows drawn on each photograph. As the inclination of the tube decreases, the bubble shape gradually changes. For angles of inclination less than $45^{\circ}$ from the horizontal, most of the liquid moves down the lower side of the tube through a crescent-shaped passage between the bubble and the tube wall. The lower side of the bubble becomes flatter as the inclination angle is decreased, and finally, near zero degrees inclination, the flow area occupies approximately the lower half of the tube and the gas bubble occupies the upper half. The dependence of propagation rate on tube inclination angle, viscosity, and surface tension is complicated. In the following work, the influence of viscosity and surface tension will be discussed, primarily for vertical tubes, and then the influence of surface tension and inclination angle will be examined for a range of parameters for which viscous effects are small.

\subsection{Viscous effects}

Previous experimental work with vertical tubes has shown that as the surfacetension parameter, $\Sigma=\left(\sigma / \Delta \rho g a^{2}\right)$, is increased, the propagation rate decreases, and that for $\Sigma \equiv 1 \cdot 2$, the rate approaches zero. However, because of limitations on values of $\sigma / \Delta \rho g$, which typically lie between 0.015 and $0.075 \mathrm{~cm}^{2}$, it is necessary to use relatively small-radius tubes in order to obtain high values of $\Sigma$. This fact, coupled with natural limitations on the minimum available values of the kinematic viscosity, implies that the Reynolds number of the flow must be reduced as $\Sigma$ is increased from 0 to $\mathbf{1 \cdot 2}$. Hence, it is not obvious that the observed reduction in bubble propagation rate is a result of increasing $\Sigma$ or decreasing $R e$, and several authors (Davies \& Taylor 1950; Goldsmith \& Mason 1962) ascribe at least part of the observed reduction to viscous effects.

However, examination of the bubble propagation-rate data presented in Hattori (1935), Dumitrescu (1943), and Goldsmith \& Mason (1962) and that obtained in the present work suggests that for Reynolds numbers greater than about 200 , the propagation rates are substantially independent of viscous effects. This result is illustrated in figure 2, where the normalized propagation rate is shown as a function of $\Sigma$ for five ranges of Reynolds number. The data are somewhat scattered, but the dependence on Reynolds number is also scattered and shows no definite trend for the whole range of $\Sigma$. Thus, these data indicate that the reduction in normalized propagation rate, which is observed as $\Sigma$ increases toward unity, is primarily a surface-tension effect and would still occur if high Reynolds number could be obtained for high $\Sigma$.

This conclusion is supported by the results of two series of tests. In the first tests, the Reynolds number of the flow was held roughly constant while $\Sigma$ was 
increased. This change was effected by using various mixtures of ethyl alcohol and water as the primary fluid in tubes of a fixed diameter. Two sets of results are given in table 1 for Reynolds numbers of roughly 700 and 200. The decrease in

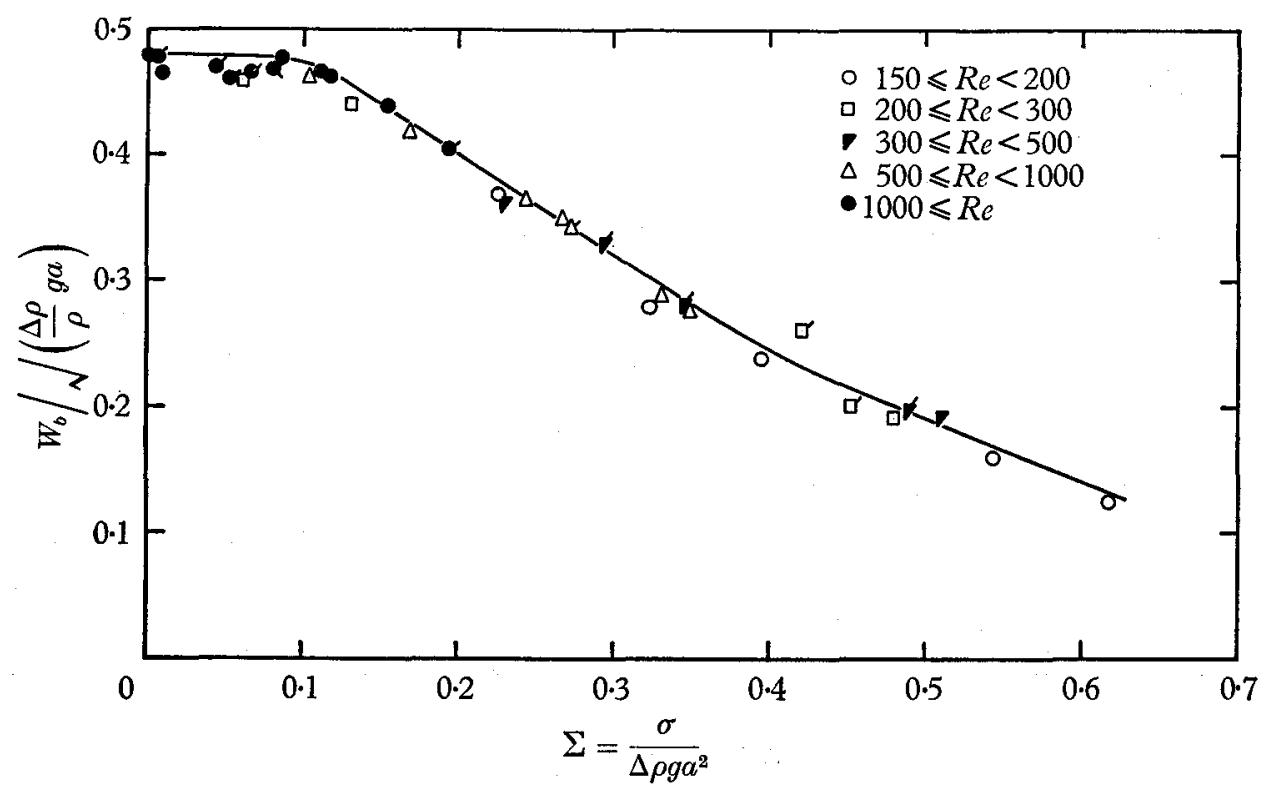

FIGURE 2. Bubble velocity vs. surface-tension parameter for ranges of Reynolds numbers. Flagged symbols from Barr (1926), Dumitrescu (1943), and Goldsmith \& Mason (1962).

normalized velocity for increasing $\Sigma$ and roughly constant Reynolds numbers is marked. For example, as $\Sigma$ is increased from 0.32 to 0.62 for the the second tube, the reduced speed decreases by more than a factor of two, from $0 \cdot 28$ to $0 \cdot 12$. Both sets of data are included in figure 2 and follow the general trend shown there. It is evident here that the surface tension has a very large effect for a fixed Reynolds number.

In the second test series, the surface-tension parameter was held roughly constant and the Reynolds number was changed over a wide range by using various mixtures of water and glycerine as the primary fluid. The effect of increasing the

$\begin{array}{ccccc}\begin{array}{c}\text { Tube } \\ \text { diameter }\end{array} & \begin{array}{c}\text { Alcohol } \\ (\mathrm{cm})\end{array} & \Sigma & & \\ 1.055 & 100 & 0 \cdot 10 & 732 & W_{b} /[(\Delta \rho / \rho) g a]^{\frac{1}{2}} \\ & 13 & 0 \cdot 17 & 637 & 0 \cdot 46 \\ & 0 & 0 \cdot 27 & 790 & 0 \cdot 35 \\ 0 \cdot 695 & 100 & 0 \cdot 23 & 302 & 0 \cdot 36 \\ & 23 & 0 \cdot 32 & 162 & 0 \cdot 28 \\ & 13 & 0 \cdot 38 & 195 & 0 \cdot 24 \\ & 0 & 0 \cdot 62 & 150 & 0 \cdot 12 \\ & & \text { TABLE } 1 & & \end{array}$


fraction of glycerine in the primary fluid is to increase the viscosity of the mixture by a large factor and to decrease the surface tension to density ratio by a small factor.

The results of these tests, shown in figure 3 , give a more complete picture of the influence of Reynolds number. Consider the curve for $2 a=0.92 \mathrm{~cm}$ shown in this figure. As the glycerine content of the fluid is increased, the velocity first increases slightly due to decreasing $\Sigma$, but then falls off rapidly due to decreasing Reynolds number. This pattern was observed for all the data shown in this figure, and the

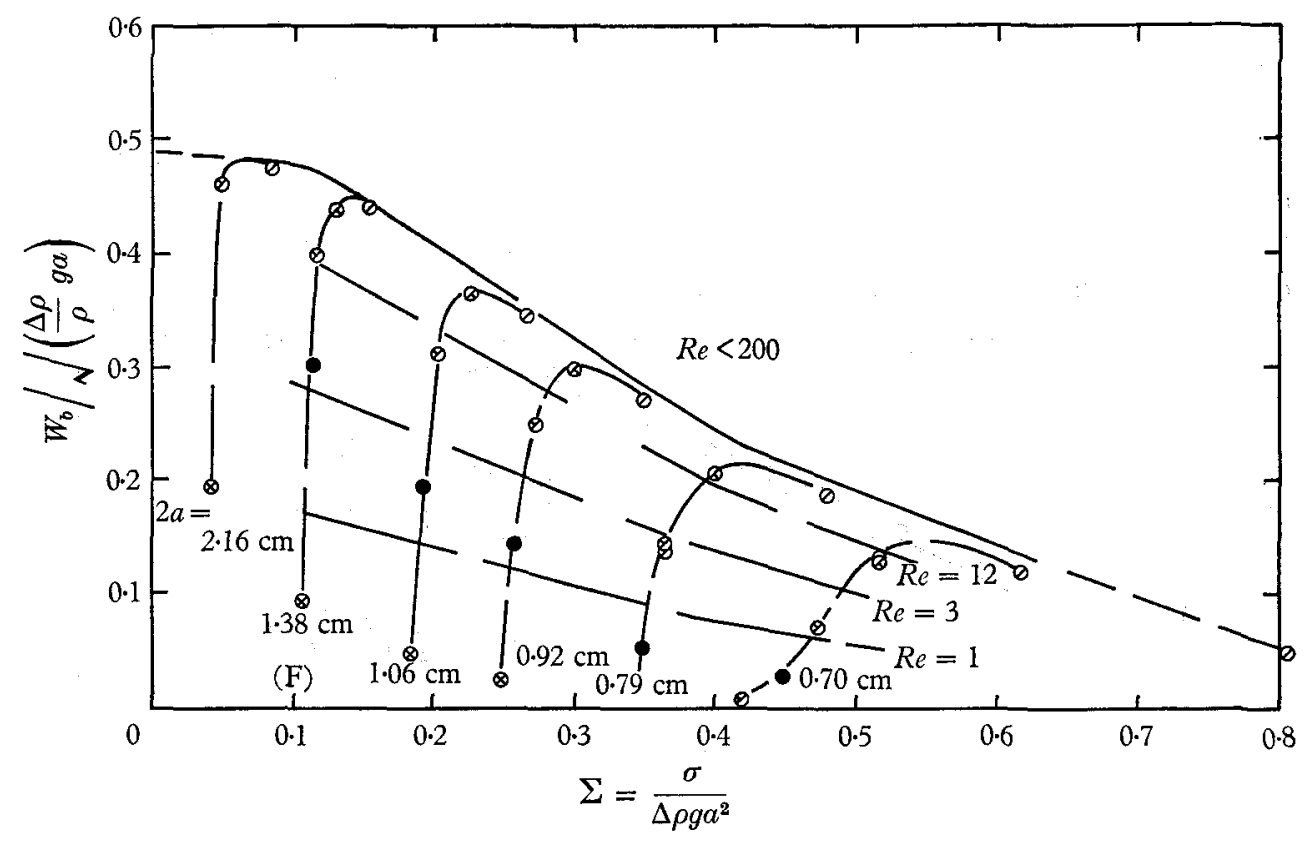

Figure 3. Influence of viscosity on bubble velocity. $\%$ glycerine by volume: $\oslash, 0 \% ; \otimes, 48 \% ; \oslash, 73 \% ; \bullet, 81 \% ; \otimes, 95 \%$.

rapid drop in normalized speed occurs for Reynolds numbers around 50 regardless of $\Sigma$. A few lines have been sketched through the data to indicate the dependence of velocity on $\Sigma$ for constant Reynolds number. These data show that as the Reynolds number is increased for a constant $\Sigma$, the propagation rate approaches a limiting value, and that for Reynolds numbers as low as 25 , the velocity is within $10 \%$ of that limiting value.

The regular variation of the curve sketched in figure 3 suggests that the influence of Reynolds number on the velocity is quite simple for $0 \leqslant \Sigma \leqslant 0 \cdot 5$. In this range of $\Sigma$, the normalized bubble velocity for a given Reynolds number and $\Sigma$ appears to be related to that at very large Reynolds number and the same $\Sigma$ by a function of the form

$$
w_{b}\{R e, \Sigma\} \approx w_{b}\{\infty, \Sigma\} f\{R e\} .
$$

In order to check this hypothesis, data of figure 3 were used to obtain a curve for $w_{b}\{\infty, \Sigma\}$. When $\Sigma<0 \cdot 2$, it was possible to obtain values of propagation rates for which $R e>1000$. These values of the Reynolds number were assumed to be 
large enough so that viscous effects were negligible and hence the experimental data gave $w_{b}\{\infty, \Sigma\}$ directly. For $\Sigma>\mathbf{0 \cdot 2}$, the maximum Reynolds numbers were less than 1000 and it was necessary to correct the data obtained at maximum Reynolds numbers. This correction was done by using equation (1) and values of $f\{R e\}$ calculated from the low $\Sigma$ data. In no case was the correction greater than $5 \%$. By this technique a value of $w_{b}\{\infty, \Sigma\}$ was obtained for each of the six curves shown in figure 3 , and the function $w_{b}\{\infty, \Sigma\}$ was obtained by drawing a smooth curve through these points.

The curve for $w_{b}\{\infty, \Sigma\}$ is shown in figure 3 and also in figure 2, with the data for $R e>150$ from the present experiments and with high-Reynolds-number data presented by other workers. The curve appears to be in reasonable agreement with these data, although there is some scatter.

Values of the function $f\{R e\}$ calculated from this curve and the data of figure 3 are shown in figure 4 . It is evident from the good correlation of the results that the relationship proposed in equation ( 1 ) is a good approximation for the range of parameters investigated in the present work.

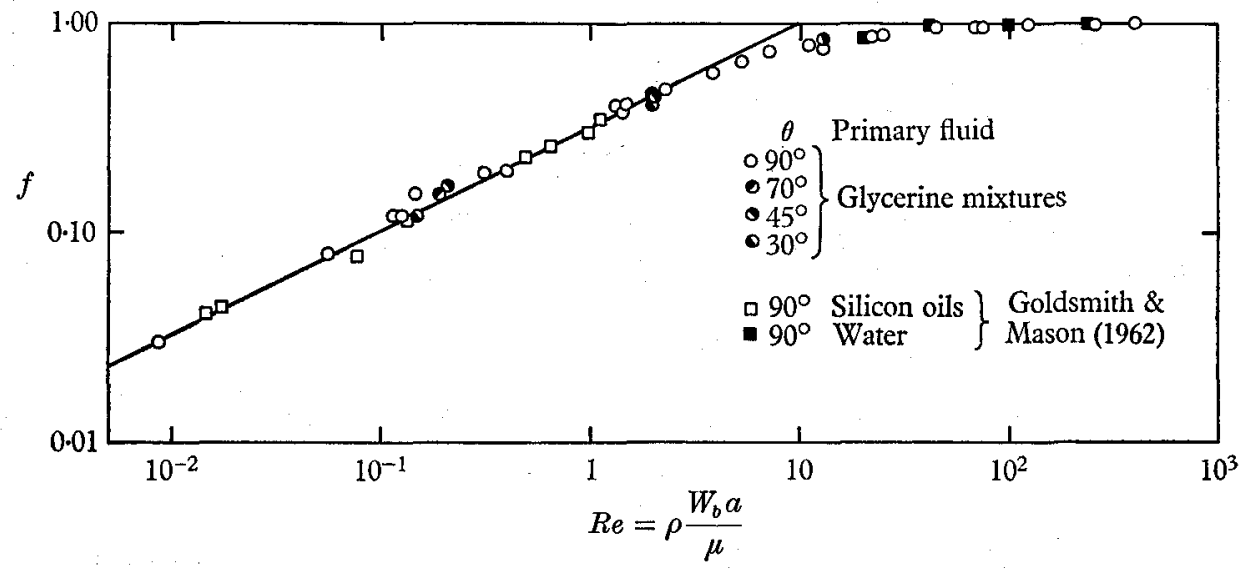

FiqURe 4. Influence of Reynolds number on the ratio, $f=W_{b}\{R e, \Sigma\} / W_{b}\{\infty, \Sigma\}$.

The tests discussed up to this point have been for vertical tubes. In addition, a few tests of this type were carried out with inclined tubes, and these data are shown as the flagged symbols in figure 4. Note that the values of the function $f\{R e\}$ were almost identical with the values obtained in the vertical tubes. Thus, these limited data suggest that the value of $f$ is independent of tube inclination angle for the range $30^{\circ} \leqslant \theta \leqslant 90^{\circ}$.

The data of figure 4 clearly fall into a high-Reynolds-number régime, $R e>100$, and a low régime, $R e<4$. In the high-Reynolds-number régime, the conclusion to be drawn from these results is that the influence of viscosity on bubble propagation rate is small and that the reduction in propagation rate as $\Sigma$ increases is primarily due to surface-tension effects.

In the low-Reynolds-number régime, the data of figure 4 show that $f$ is proportional to the square root of the Reynolds number, and consequently, equation (1) can be rewritten as

$$
\mu W_{b} /\left(\Delta \rho g a^{2}\right) \propto w_{b}^{2}\{\infty, \Sigma\}
$$


When flows with constant values of $\Sigma$ are considered, this equation reduces to

$$
\mu W_{b} /\left(\Delta \rho g a^{2}\right)=\text { const. }
$$

This result was recognized by early experimenters and was the basis for the Cochius viscosimeter. Note that when $\Sigma$ is less than $0 \cdot 1, W_{b}\{\infty, \Sigma\}$ becomes independent of the surface-tension parameter, and in this range equation (2) reduces to equation (3). Consequently, the Cochius viscosimeter should be quite accurate as long as $0 \cdot 1>\Sigma$ and $R e<1 \cdot 0$.

Goldsmith \& Mason have also obtained data on bubble velocity in the lowReynolds-number régime. Their data were obtained with silicon oil-air bubbles, and covered the ranges $2 \times 10^{-5} \leqslant R e \leqslant 1$ and $0 \cdot 15 \leqslant \Sigma \leqslant 0 \cdot 8$. Values of $f$ calculated from their results by the author are shown in figure 4 . The agreement is good over the Reynolds-number range of the present experiments, and an extension of the straight line in the figure to $R e=10^{-5}$ correlates the remainder of their data equally well.

Goldsmith \& Mason correlated their data by a function of the form

$$
\mu W_{b} /\left(\Delta \rho g a^{2}\right)=k\{\Sigma\} \quad(R e<1)
$$

which was suggested by Barr. The present correlation, given in equation (2), is identical to equation (4) for the low-Reynolds-number régime, but has the virtue of covering the high and intermediate as well as the low range of Reynolds numbers.

In addition to bubble velocity, some information concerning bubble geometry is available for the low-Reynolds-number régime. Examination of photographs of bubbles rising through viscous fluids shows that the thickness of the fluid film between the bubble and the tube wall reaches a constant value within a few tube diameters of the bubble nose when the Reynolds number is less than one. Goldsmith \& Mason made a careful study of the dependence of this constant film thickness on the Reynolds number and surface tension. These investigators discovered the remarkable fact that the film thickness is independent of the Reynolds number, and hence of the bubble speed, for the range $10^{-5} \leqslant R e \leqslant 1$. They also found that the thickness is a sensitive function of the surface tension. Further analysis of their data shows that the film thickness $\delta$, normalized by the tube radius, is a simple function of $\Sigma$

$$
\delta / a \approx \frac{1}{3}(1-\Sigma / 0 \cdot 95)
$$

The only exceptions to this dependence were found when data were examined for fluids to which surface-active ingredients had been added. It is the opinion of the author that data obtained with these fluids disagreed with those obtained with homogeneous materials because the dynamic manner in which the interface is formed at the bubble nose prevents the uniform action of the surface-active agents.

Several film thicknesses were also measured from photographs taken during the present tests. They agree with equation (5) as long as $R e<2$. However, for $R e>2$, the thickness decreases rapidly with increasing Reynolds number.

Given the fact that film thickness is independent of Reynolds number, the results of equation (3) can be derived directly from the continuity equation; such 
a development is given in Goldsmith \& Mason (1962). Although the results given by equations (1), (3), and (5) suggest an underlying simplicity for the flow process in the high- and low-Reynolds-number régimes, no analytic explanation for these results has been found.

\subsection{Inclination angles}

The effect of inclination angle on bubble propagation rate is complex because of the change in bubble geometry which occurs in response to changes in inclination angle. These geometric changes are illustrated in figure 1 (plate 1).

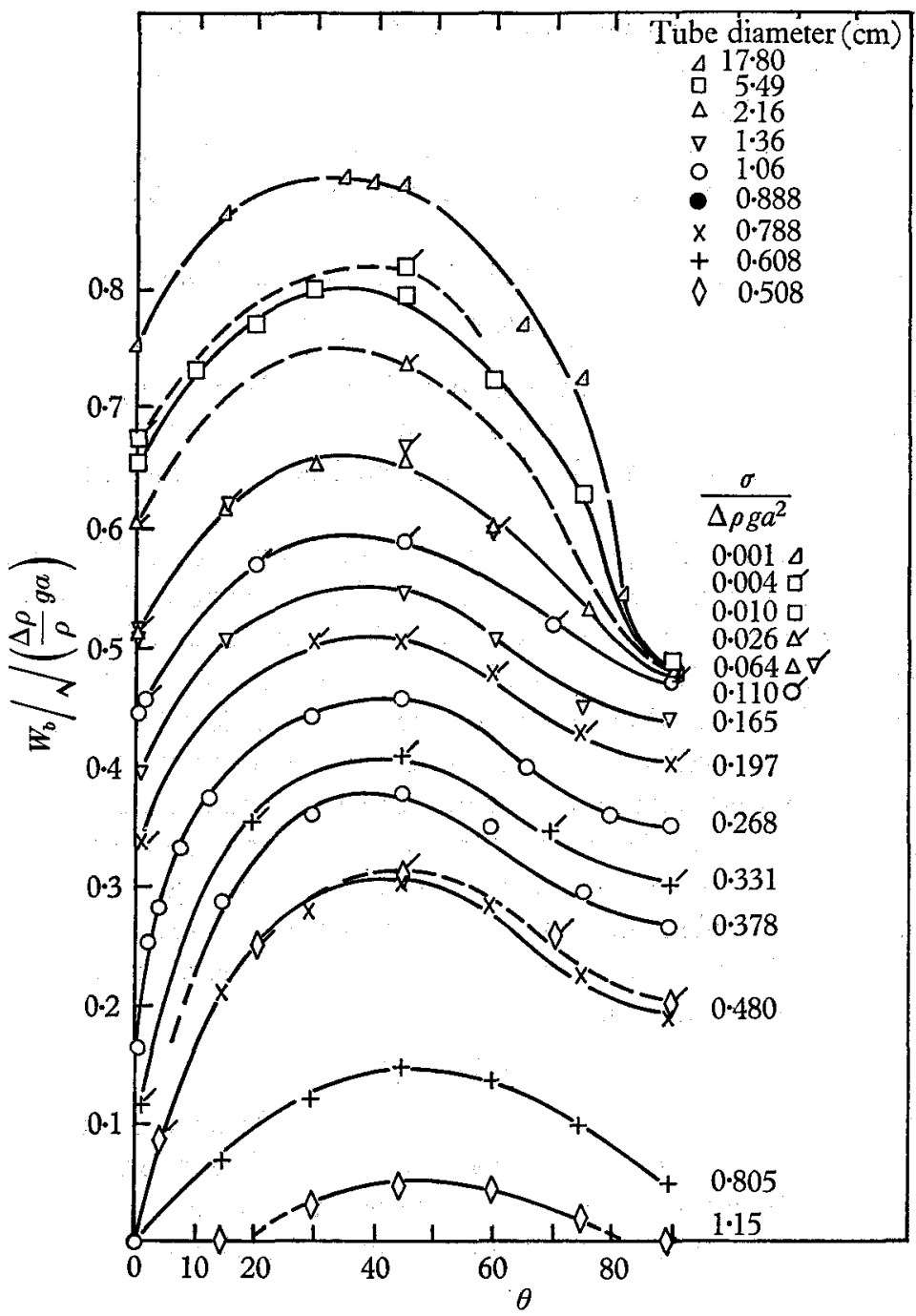

FrGURE 5. Variation of normalized velocity with inclination angle, liquid-air system. Air bubble moving into fluid: unflagged symbols-water; flagged symbols - acetone.

The bubble propagation rates obtained for a number of tubes and fluids, covering a wide range of $\Sigma$ and $R e_{0}$, are shown in figures 5 and 6 , where the normalized velocity is given as a function of the inclination angle $\theta$, measured from the 
horizontal position. Values of $\Sigma$ corresponding to each curve are shown at the right-hand side of the figure. In figure 5, air was the light material, and data were obtained with water and acetone (the flagged symbols) as the primary fluid. In figure 6, two dense materials were used as noted, and some air-liquid data are shown for reference purposes. Viscous effects are not important for

$$
W_{b} / \sqrt{ }\{(\Delta \rho / \rho) g a\}>0 \cdot 15
$$

since in this régime $R e>200$.

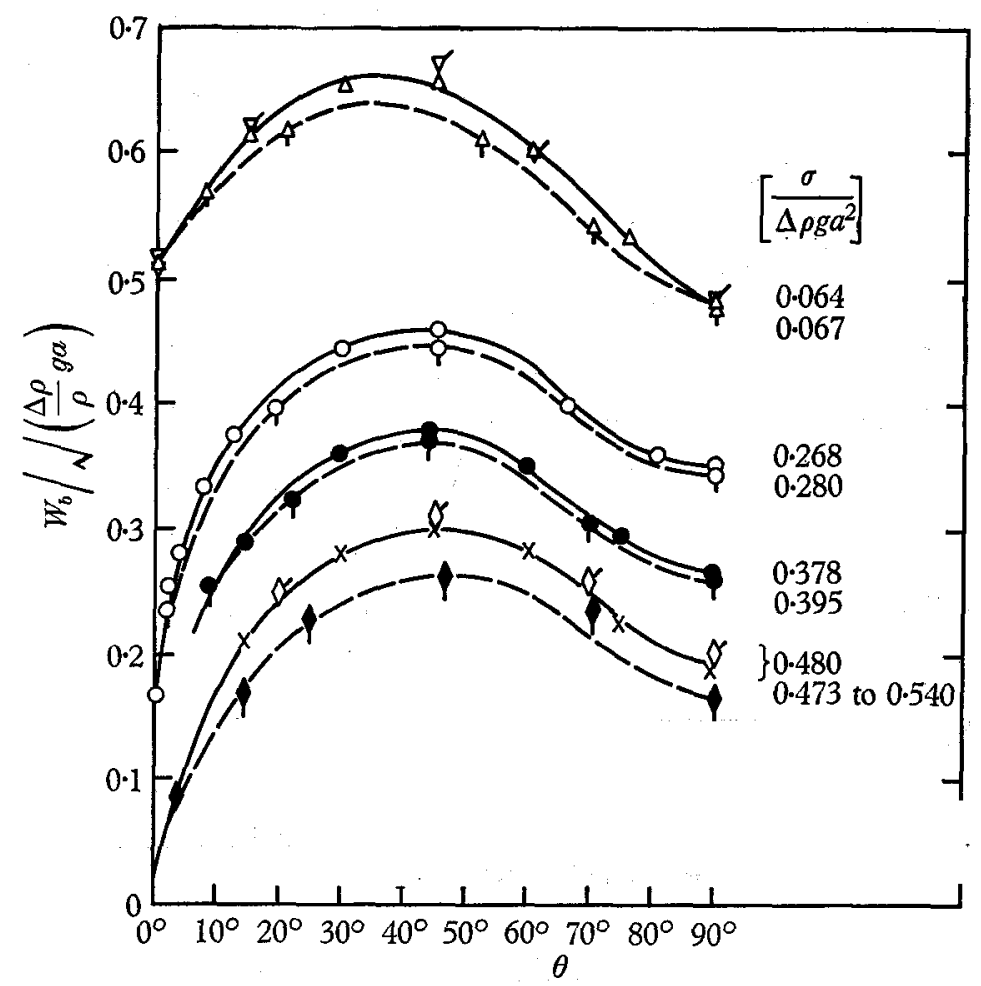

FIGURE 6. Variation of normalized velocity with inclination angle, liquid-liquid system.

\begin{tabular}{ccc} 
& $\begin{array}{c}\text { Tube } \\
\text { diam. }\end{array}$ & Bubble moving \\
& (cm) & into fluid \\
$\Delta$ & 2.16 & Air- $\mathrm{H}_{2} \mathrm{O}$ \\
$\nabla$ & $1 \cdot 38$ & Air-acetone \\
\hline & $2 \cdot 16$ & $\mathrm{CCl}_{4}-\mathrm{H}_{2} \mathrm{O}$ \\
\hline & $1 \cdot 06$ & $\mathrm{Air}_{2}-\mathrm{H}_{2} \mathrm{O}$ \\
\hline & $1 \cdot 06$ & $\mathrm{CCl}_{4}-\mathrm{H}_{2} \mathrm{O}$
\end{tabular}

$\begin{array}{ccc} & \text { Tube } & \\ & \text { liam. } & \text { 3ubble moving } \\ & (\mathrm{cm}) & \text { into fluid } \\ - & 0.888 & \mathrm{Air}-\mathrm{H}_{2} \mathrm{O} \\ & 0.888 & \mathrm{CCl}_{4}-\mathrm{H}_{2} \mathrm{O} \\ \times & 0.788 & \mathrm{Air}^{-}-\mathrm{H}_{2} \mathrm{O} \\ 5 & \mathbf{0 . 5 0 8} & \mathrm{Air}-\text { acetone } \\ & 0.508 & \mathrm{Hg}-\mathrm{H}_{2} \mathrm{O}\end{array}$

In general, the propagation rate of the bubbles increases to a maximum value as the inclination angle decreases from the vertical position to $45^{\circ}$, and a further reduction in angle causes the rate to decrease. In all cases for which the normalized speed was greater than $0 \cdot 1$, steady propagation rates were obtained for inclination angles from $90^{\circ}$ down to a few degrees. However, for the horizontal position, i.e. $\theta \equiv 0^{\circ}$, a steady propagation rate was only observed if $\Sigma<0 \cdot 1$, and 
none was found for larger values of $\Sigma$. The latter result does not appear to be a viscous effect.

All propagation rates increase with decreasing $\Sigma$. However, the propagation rate in the vertical tube appears to approach within a few per cent of its limiting value for $\Sigma \approx 0 \cdot 1$, whereas for other inclinations the propagation rates continue to increase rapidly as $\Sigma$ approaches zero.

Certain features of the dependence of the propagation rate on inclination angle described above are of sufficient interest to warrant further discussion. First; consider the fact that as $\Sigma$ approaches zero, the normalized propagation rate in

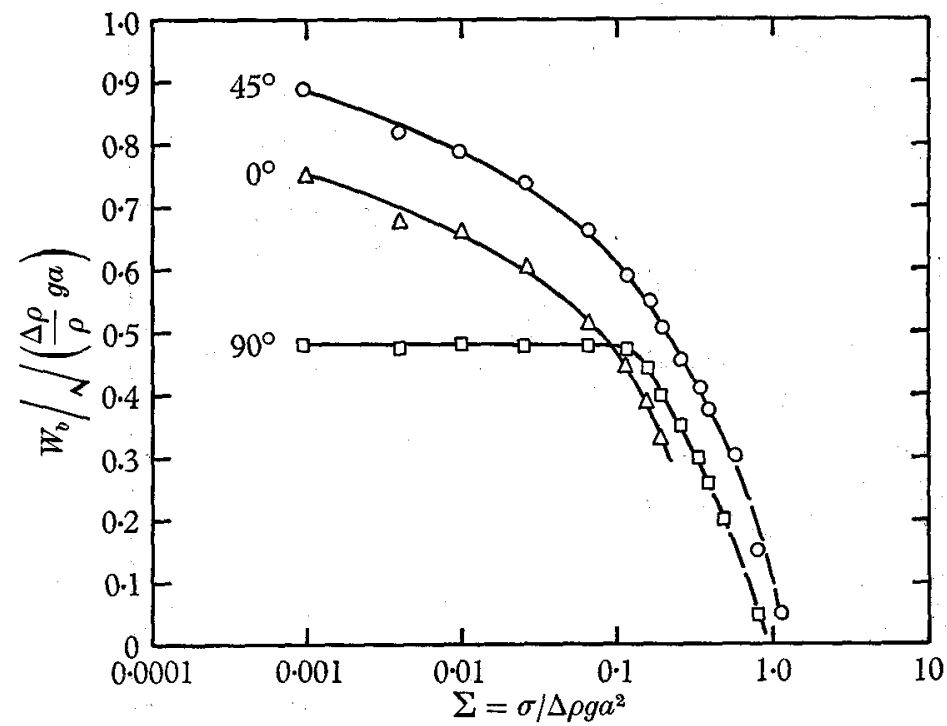

FrguRE 7. Variation of normalized velocity with surface-tension parameter for $\theta=0^{\circ}, 45^{\circ}$, and $90^{\circ}$.

a vertical tube, $\theta=90^{\circ}$, approaches a limiting value, whereas that for other inclination angles continues to increase. This dependence is more clearly shown by the plot of normalized speed versus $\ln \Sigma$ given in figure 7 for inclination angles of $90^{\circ}, 45^{\circ}$, and $0^{\circ}$. It is quite clear here that the propagation rates in a vertical tube approach a limiting value as $\Sigma$ approaches zero, but that neither the $0^{\circ}$ or $45^{\circ}$ data do so in the range investigated.

Examination of a large number of photographs taken over the whole range of $\theta$ and $\Sigma$ suggests an explanation for this result. For the vertical tube, the radius of curvature at the nose of the bubble was found to decrease slightly as $\Sigma$ was decreased to $0 \cdot 10$; for smaller $\Sigma$, the radius was almost constant. However, for $\theta=45^{\circ}$ and $0^{\circ}$, a much more dramatic change occurs. For these inclination angles, the radius of curvature decreases by about a factor of two as $\Sigma$ is decreased from 1 to $0 \cdot 1$. However, a further reduction in $\Sigma$ continues to reduce the curvature and at $\Sigma=10^{-3}$, the radius of curvature is less than $0 \cdot 05 a$.

The photographs shown in figure 8 (plate 2) illustrate this change for tubes at $45^{\circ}$ inclination angle. Here the values of $\Sigma$ change from 0.2 to 0.001 and the nose 
radius changes from an appreciable fraction of the tube radius to a very small fraction of it. These results suggest that in the limit, as $\Sigma$ approaches zero, the radius of curvature at the stagnation point also approaches zero for non-vertical tubes. Since the theoretical treatments of Dumitrescu and of Davies \& Taylor have shown that the radius of curvature at the stagnation point is critical in fixing the bubble velocity in a vertical tube, one would expect that the propagation rate for inclined tubes would also continue to change as $\Sigma$ approaches zero in response to the continued change in the ratio of the radius of curvature to the tube radius. Thus, the differences between the limiting values of the radius of curvature near the stagnation point for bubbles with $\theta=90^{\circ}$ and $0 \leqslant \theta<90^{\circ}$ may explain the differences in behaviour of the normalized velocities shown in figures 5, 6, and 7 . The vertical tube appears to be a singular case in the limit of very small $\Sigma$.

A second interesting feature of the data presented in figure 5 is the behaviour of the normalized velocity for horizontal tubes. The reason for the non-steady flows observed when the normalized speed is less than 0.5 is connected with the flow speed in the fluid passing under the bubble near the tube exit. If this velocity is less than half the critical speed for the flow, disturbances originating at the exit can overtake the bubble and no steady flow can be established. However, when this exit velocity is greater than half the critical speed, disturbances from the exit move upstream slower than the bubble and hence steady motion can be maintained.

Under most conditions tested, the fluid occupies roughly the lower half of the tube for the horizontal position, and consequently the critical speed is very roughly $\sqrt{ }(g a)$. For this level of fluid, continuity arguments show that the exit and bubble speeds are equal. Therefore both must be half the critical speed in the limiting case for steady motion. This limit is in good agreement with that shown by the data of figure 5, which indicate that the limit lies at a velocity between $0 \cdot 44$ and $0.52 \sqrt{ }(g a)$.

However, some restrictions on the interpretation of this data should be emphasized here. The stable bubbles were obtained in relatively large-diameter tubes with length-to-diameter ratios in the range of $50: 1$ to $20: 1$. For these tubes, the length of the flow from the bubble to the exit was short enough that viscous effects were unimportant. If the length of the flow between the bubble nose and the tube exit is increased indefinitely, then it is evident that viscous effects will slow the efflux and produce a reduction in the bubble propagation rate. Hence, the zeroinclination-angle data represent a rather special flow situation and may be regarded as the limiting velocity as $\theta$ approaches zero.

A third point of interest concerns the comparison of the data obtained with two liquids to that obtained with liquid-gas systems. These data are presented in figure 6 , and in each case it was possible to compare data from liquid-liquid systems with that from a liquid-gas system which had very nearly the same value of surface-tension parameter. The data agree both qualitatively and quantitatively, with the possible exception of the mercury-water system. Quantitative comparison of other results with the mercury-water system data was not possible, because the well-known uncertainties in the evaluation of the interfacial tension 
between mercury and water cause difficulty in obtaining a precise value of $\Sigma$ for this system. The good correlation of the data shown in figures 5 and 6 indicates that the scaling procedures used for velocity and surface tension are correct.

In studying the liquid-liquid systems, an instability in the interface was encountered for most of the conditions investigated. This instability was in the form of wave-like disturbances which grew in amplitude as the distance downstream of the bubble nose was increased. Two types of distortions were observed. In nearly vertical tubes, the waves were predominantly axisymmetric and the

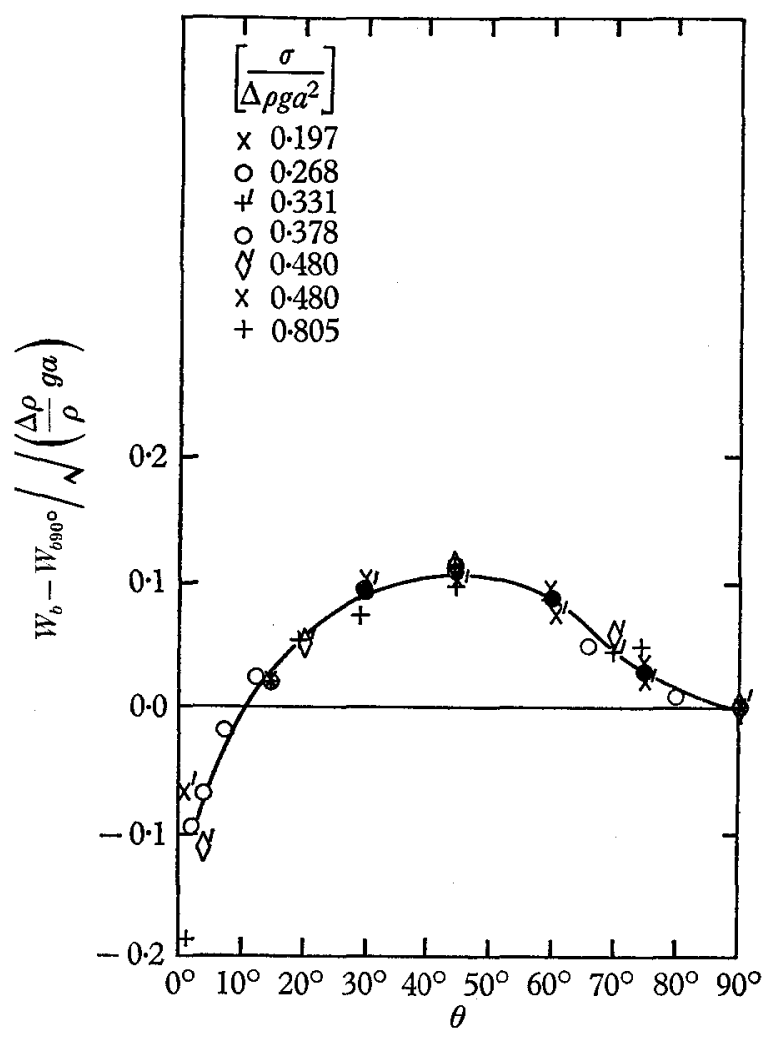

FIGURE 9. Variation of the normalized velocity difference with inclination angle.

wavelength was small compared with the tube radius. In nearly horizontal tubes, the wave length was of the order of the tube radius, and the amplitude of the wave increased rapidly with bubble velocity for a given set of fluids. However, the good agreement between liquid-liquid system data and liquid-air data indicates that the instability did not produce a change in the velocity as long as large-amplitude waves were restricted to positions at least one tube radius downstream of the bubble nose. The instability is thought to be of the Helmholtz type, modified by the influence of the gravitational field, which is not perpendicular to the interface.

One of the surprising features of the data presented in figures 5 and 6 is the form of the dependence of the propagation velocity on inclination angle. For the range $0 \cdot 1 \leqslant \Sigma \leqslant 0 \cdot 8$, and $\theta>15^{\circ}$, the dependence can be put in the form

$$
w_{b}\{\Sigma, \theta\}=w_{b}\left\{\Sigma, 90^{\circ}\right\}+g\{\theta\} .
$$


This result can be seen by inspection of the data and is also shown by a plot of the function $g\{\theta\}$ shown in figure 9 . A similar result is obtained for the ranges $\Sigma<0 \cdot 1$ and $\theta<50^{\circ}$.

\subsection{Stability of bubble geometry and variant forms}

In almost all of the present experiments, the bubble shapes illustrated in figure 1 (plate 1) were found to be the stable shapes of the bubbles. Extreme agitation of the tube and a wide range of techniques used to initiate bubble formation could lead momentarily to a different bubble geometry; however, the bubble geometry almost always reverted to these standard forms.

Three exceptions to the standard bubble geometry were noted. The first of these is the instability in the interface which has been discussed above. This disturbance appears to be of the Helmholtz type rather than the Taylor or capillary forms. Analysis is complicated because of the inclination of the interface with respect to the gravitational acceleration, the acceleration of the streams, and the finite and changing depth of the streams. However, it is clear that the amplitude of the disturbance grows with increasing bubble velocity and with decreasing values of the density ratio of the heavy to the light fluid.

The second exception has to do with the turbulent level and flow disturbance in the tubes. It was found that the bubble geometry and propagation rate were sensitive functions of any flow disturbances in the tube. For example, if two bubbles are allowed to pass through the tube one after another, and if the distance between the bubbles is small enough, flow disturbances in the wake of the first bubble can cause large-scale distortions of the second bubble. The net effect of these disturbances is often to cause an increase in the velocity of the second bubble which can result in the coalescence of the bubbles. As a second example, consider a vertical tube, full of fluid, in which a circulation has been set up by allowing a small flow of air to leak into the tube. When a bubble under study reaches the region of circulation, its shape is again radically distorted by the local flow field, and the bubble propagation rate can be greatly enhanced. This behaviour is to be expected, since the bubble propagation rates are small, and both methods of disturbing the flow mentioned here produce disturbance velocities as large as, or larger than, the propagation rate of the bubble.

The third exception to be discussed concerns the influence of contact angle at the tube wall on the bubble geometry for vertical tubes. When liquid-air-tube systems are used in which the contact angle is $90^{\circ}$ or greater, a standard stable bubble configuration is observed which is different from the axisymmetric shape. This shape is illustrated by the drawings shown in figure 10. Here, the gas-liquid interface is in the shape of a cap of large radius which makes contact with the wall at angles somewhat less than $90^{\circ}$; on one side of the cap, the liquid flows down the side of the tube in a narrow stream. This bubble shape was stable for air or water bubbles moving through mercury in a glass tube and for air bubbles moving through water in Plexiglas tubes. In each case, the contact angle in the primary fluid at the interface between the fluid and the wall was large; $90^{\circ}$ for the waterair-Plexiglas system and $180^{\circ}$ for the mercury-water-glass and mercury-airglass systems. Note that in the case of a mercury bubble falling through water, 
water is the primary fluid and the contact angle in the water is zero. For the latter case, the normal bubble shape is observed. This contrast emphasizes the result that the bubble shape depends on the contact angle in the primary fluid.

The stability limits for this form of bubble are connected with the motion of a film of the primary fluid at the point of contact of the cap and the wall. The important mechanism for fixing the stable shape is apparently that which fixes the rate at which this thin liquid film left on the tube wall by the motion of the bubble can contract toward the moving bubble. For small diameters, and hence small bubble velocity, this contraction rate can be equal to the propagation rate of the fluid, and consequently, the bubble-cap shape can be maintained. When
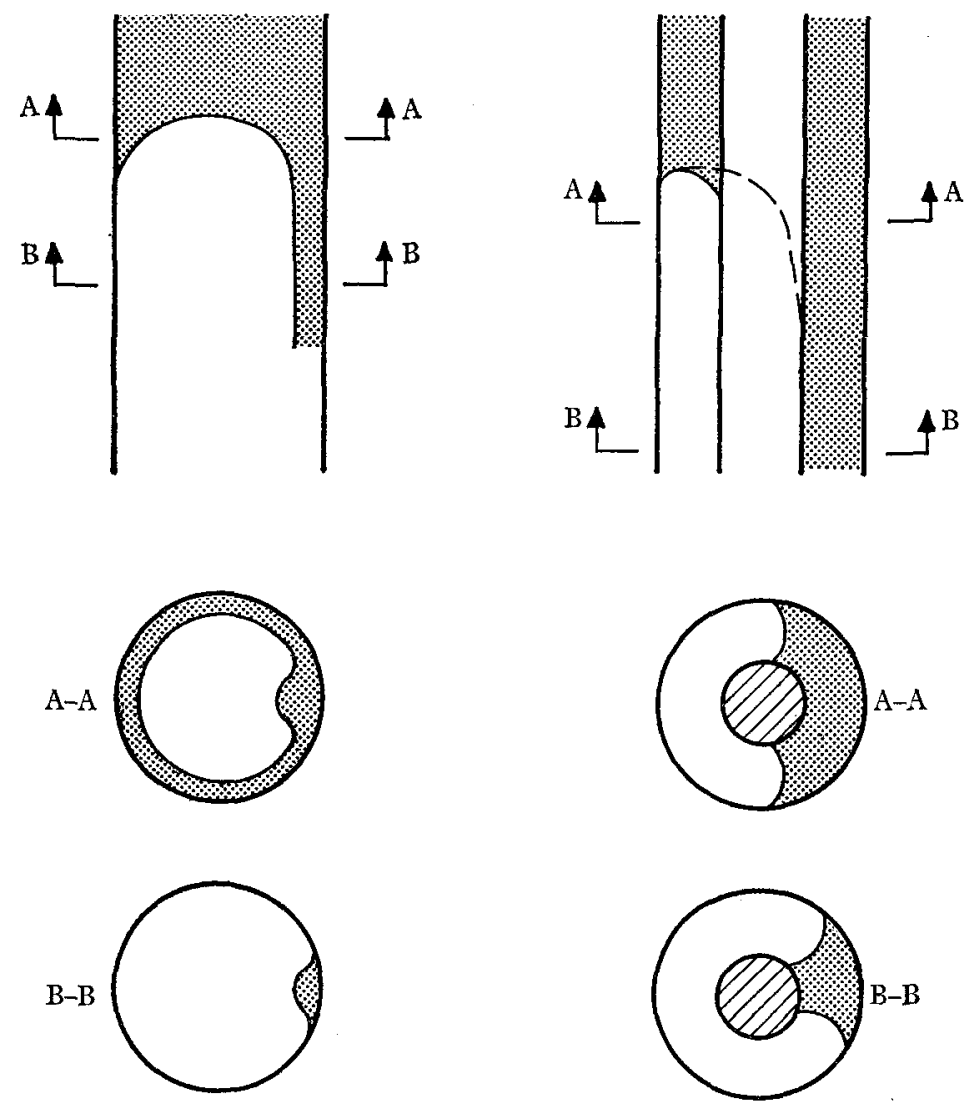

Figure 10. Two variant bubble shapes.

the tube diameter is increased, the bubble propagation rate increases; whereas in contrast, the film propagation rate is apparently constant. Thus, for large enough tubes, the film is left behind the bubble cap. As the film is left farther and farther behind the cap, it gradually thickens in the neighbourhood of the cap, and finally causes a transition from the bubble cap to the usual axisymmetric geometry. Transitions of this type have not been observed for the small (up to $1 \mathrm{~cm}$ diameter) glass tubes used in the mercury experiments. 
A second bubble shape was also investigated briefly as a result of conversations with D. Gluck and J. Gille of North American Aviation, Downey, California, who reported a second unsymmetric bubble shape. Consider the emptying of a space between annular cylinders. For this geometry, the bubble geometry is again non-symmetric with the fluid flowing down in a restricted region on one side of the annular area and the gas moving up in the rest of the annulus. For large ratios of the outer to inner radius of the annulus, the width of the fluid flow area near the bubble nose is approximately given by the diameter of the inner tube, see figure 10.

The propagation rate for the two unsymmetric bubbles discussed here has been examined for a few cases. The rates appear to be close to those reported for the symmetric bubble in a tube of the same diameter.

\section{Conclusions}

Experimental studies of the motion of long bubbles in tubes have led to a better understanding of the influence of surface tension and viscosity on bubble propagation in vertical tubes. In addition, the influence of tube inclination angle has been described and qualitative explanations for certain features of the flow have been suggested.

\section{REFERENCES}

BARR, G. 1926 Phil. Mag. 1, 395.

Davies, R. M. \& TaYloR, Sir G. I. 1950 Proc. Roy. Soc. A, 200, 375.

Dumitresou, D. T. 1943 Z. angew. Math. Mech. 23, 139.

Goldsmith, H. L. \& Mason, S. G. 1962 J. Fluid Mech. 14, 42.

HaRmathy, T. Z. 1960 Amer. Inst. of Chem. Engng J. 6, 281.

Hattori, S. 1935 Rept. Aeronaut. Res. Inst., Tokyo Imp. Univ. no. 115. 